\title{
Pleiotropic Effects of Obesity on Fracture Risk: The Study of Women's Health Across the Nation
}

\author{
Shinya Ishii, ${ }^{1}$ Jane A Cauley, ${ }^{2}$ Gail A Greendale, ${ }^{3}$ Carrie Nielsen, ${ }^{4}$ Carrie Karvonen-Gutierrez, ${ }^{5}$ \\ Kristine Ruppert, ${ }^{2}$ and Arun S Karlamangla ${ }^{3}$ \\ ${ }^{1}$ Department of Geriatric Medicine, Graduate School of Medicine, University of Tokyo, Bunkyo-ku, Tokyo, Japan \\ ${ }^{2}$ Department of Epidemiology, Graduate School of Public Health, University of Pittsburgh, Pittsburgh, PA, USA \\ ${ }^{3}$ Department of Medicine/Division of Geriatrics, David Geffen School of Medicine at UCLA, Los Angeles, CA, USA \\ ${ }^{4}$ School of Medicine, Oregon Health and Science University, CR113, Portland, OR, USA \\ ${ }^{5}$ School of Public Health, University of Michigan, Ann Arbor, MI, USA
}

\begin{abstract}
Some aspects of an obese body habitus may protect against fracture risk (higher bone mineral density [BMD] and greater tissue padding), while others may augment that risk (greater impact forces during a fall). To examine these competing pathways, we analyzed data from a multisite, multiethnic cohort of 1924 women, premenopausal or early perimenopausal at baseline. Obesity was defined as baseline body mass index $(\mathrm{BMI})>30 \mathrm{~kg} / \mathrm{m}^{2}$. Composite indices of femoral neck strength relative to fall impact forces were constructed from DXA-derived bone size, BMD and body size. Incident fractures were ascertained annually during a median follow-up of 9 years. In multivariable linear regression adjusted for covariates, higher BMI was associated with higher BMD but with lower composite strength indices, suggesting that although BMD increases with greater skeletal loading, the increase is not sufficient to compensate for the increase in fall impact forces. During the follow-up, 201 women had fractures. In Cox proportional hazard analyses, obesity was associated with increased fracture hazard adjusted for BMD, consistent with greater fall impact forces in obese individuals. Adjusted for composite indices of femoral neck strength relative to fall impact forces, obesity was associated with decreased fracture hazard, consistent with a protective effect of soft tissue padding. Further adjustment for hip circumference, a surrogate marker of soft tissue padding, attenuated the obesity-fracture association. Our findings support that there are at least three major mechanisms by which obesity influences fracture risk: increased BMD in response to greater skeletal loading, increased impact forces, and greater absorption of impact forces by soft tissue padding. ( $) 2014$ American Society for Bone and Mineral Research.
\end{abstract}

KEY WORDS: OBESITY; OSTEOPOROSIS; FRACTURE RISK; STRENGTH RELATIVE TO LOAD; FALL IMPACT FORCES; SOFT TISSUE PADDING

\section{Introduction}

$\mathrm{O}$ besity has long been thought to protect against osteoporosis ${ }^{(1)}$ and fragility fractures, ${ }^{(2)}$ primarily because greater skeletal loading in obese individuals leads to increased bone mineral density $(\mathrm{BMD})^{(2,3)}$ and more favorable bone geometry. ${ }^{(4,5)}$ However, impact forces in a fall are also greater in obese individuals because body weight is greater. Thus, for obesity to reduce fracture risk, the increase in BMD stimulated by greater skeletal loading has to compensate for greater impact forces. Unfortunately, both chronic inflammation and type 2 diabetes are also more prevalent in obese individuals, and both have deleterious influences on fracture risk; ${ }^{(6-9)}$ therefore the BMD advantage from greater skeletal loading may not be sufficient to reduce one's fracture risk.

In fact, multiple recent studies report that fractures are no less common in obese individuals than in the non-obese, ${ }^{(10,11)}$ and that fracture risk in some body sites (eg, extremities) is actually increased with obesity, ${ }^{(11-15)}$ challenging the conventional assumption that obesity protects against fractures. There does, however, appear to be some protection conferred by obesity against fractures of the hip and pelvis in these studies. ${ }^{(12-15)}$ Greater absorption of impact forces by soft tissue padding around the hip may underlie this relative reduction in hip and pelvic fracture risk in obese women; ${ }^{(4,11,12,14)}$ however, the role of soft tissue padding in obesity-fracture associations has not been empirically examined in longitudinal studies.

To disentangle these disparate effects of obesity and fracture risk, we tested a series of hypotheses aimed at isolating different components in the obesity-fracture relationship. We hypothesized that:

1. Obesity would be associated with increased BMD, reflecting the increased bone mass stimulated by greater skeletal loading. 
2. Obesity would be associated with reduced bone strength relative to load (ie, that the BMD benefit of obesity would be insufficient to compensate for greater fall impact forces).

3. Adjusted for BMD (thus, removing the effects of body weight on BMD), obesity would be associated with increased fracture risk, reflecting greater fall impact forces (ie, we hypothesized that, of the remaining pathways, the influence of body weight on fall impact forces would dominate).

4. Adjusted for bone strength relative to load (thus, removing the effects of body weight on both BMD and fall impact forces), obesity would be associated with decreased fracture risk, reflecting the protective role of soft tissue padding (ie, we hypothesized that, of the remaining pathways, the protection provided by soft tissue padding would dominate).

We used data from the Hip Strength Across the Menopause Transition study to test these hypotheses.

\section{Materials and Methods}

\section{Study design and population}

The Study of Women's Health Across the Nation (SWAN) is a multicenter, multiethnic longitudinal study to characterize the biological and psychosocial changes that occur during the menopausal transition. Between 1995 and 1997, a screening survey to assess study eligibility was conducted in women using either community-based or population-based sampling frames at each of seven participating clinical sites. ${ }^{(16)}$ Briefly, cohort entry criteria were as follows: age 42 to 53 years, with intact uterus and at least one ovary, not using sex steroid hormones at enrollment, had at least one menstrual period in the 3 months prior to screening, and self-identified as either white, African American, Hispanic, Chinese, or Japanese. Each site recruited at least 450 eligible women into the cohort in 1996 and 1997, resulting in an inception cohort of 3302 women. ${ }^{(17,18)}$

Five sites (Boston, Detroit, Pittsburgh, Los Angeles, and Oakland) collected DXA scans of the hip and lumbar spine in all but 46 participants who weighed more than $136 \mathrm{~kg}$ (the scanner weight limit); they constituted the SWAN bone cohort. All five sites enrolled whites, and each site also enrolled women belonging to one prespecified minority ethnic group: African American in Boston, Detroit, and Pittsburgh and Japanese and Chinese in Los Angeles and Oakland, respectively. The Hip Strength Across the Menopause Transition study, the focus of this report, measured femoral neck size using archived hip DXA scans from the 1960 women in the SWAN bone cohort who had a baseline and two or more follow-up scans by follow-up visit 10 (2006-2007). At baseline, 36 women did not get either bone size or body size measurements, leaving 1924 women in the analytic sample (963 white, 503 African American, 238 Japanese, and 220 Chinese). All protocols were approved by Institutional Review Boards at each site and all participants gave written informed consent.

\section{Assessment of BMD and bone strength relative to load}

Using the OsteoDyne Hip Positioner System (Osteodyne Inc.), DXA scans of the posterior-anterior lumbar spine and total hip were acquired at the baseline visit. Hologic QDR 4500 models were used in Boston, Detroit, and Los Angeles, and QDR 2000 scanners were used in Pittsburgh and Oakland (Hologic Inc., Waltham, MA, USA). A standard quality-control program, conducted in collaboration with Synarc, Inc. (Newark, CA, USA), included daily phantom measurements, 6-month cross-calibration with a circulating anthropomorphic spine standard, local site review of all scans, central review of scans that met problemflagging criteria, and central review of $5 \%$ random sample of scans. The 2D-projected (areal) BMD in the femoral neck and lumbar spine were recorded.

As markers of relative bone strength, we employed composite indices of femoral neck strength relative to load that integrate BMD, bone size, and (in light of the "supply and demand balance") body size. ${ }^{(19)}$ They have been shown to predict fracture risk in white women ${ }^{(19)}$ and in women from a multi-ethnic cohort, ${ }^{(20)}$ and unlike $\mathrm{BMD}$, they do not require race/ethnicity information to do so. ${ }^{(20)}$ Also unlike BMD, differences in the composite strength indices between diabetics and nondiabetics are consistent with known differences in fracture risks between these groups. ${ }^{(7)}$ In addition, unlike BMD, the composite strength indices are inversely associated with serum C-reactive protein (CRP) levels, a marker of chronic inflammation, and partially explain the increased fracture risk associated with inflammation. ${ }^{(6)}$

Two bone-size measurements were made on archived baseline hip scans using pixel dimensions provided by the manufacturer: femoral neck axis length (FNAL) and femoral neck width (FNW) (Fig. 1). The composite indices of femoral neck strength relative to load were computed using height, weight, FNAL, FNW, and femoral neck BMD (Fig. 1). ${ }^{(19)}$ Compression strength index $(\mathrm{CSI})$ reflects the ability of the femoral neck to withstand axial compressive loads proportional to body weight, bending strength index (BSI) reflects its ability to withstand bending forces proportional to body weight, and impact strength index (ISI) reflects its ability to absorb the energy of impact in a fall from standing height (which is proportional to the product of body height and weight). ${ }^{(19)}$ To examine reproducibility of the composite strength indices, 20 women were scanned twice after repositioning; the intraclass correlation coefficient for each index was greater than 0.98 .

\section{Incident fracture ascertainment and classification}

During each of nine annual follow-up visits, fractures since the previous visit were self-reported using a standardized interviewer-administered questionnaire. In all years, the number of fractures, body site(s), and how fractures occurred were recorded. SWAN initiated collection of the date of fracture at follow-up visit 6 . Because dates of fractures were not collected in the first six follow-ups, they were imputed using the midpoint between the participant's index and previous visits. Fractures reported at visit 6 and later were confirmed by reviewing medical records. Medical records were available for $85 \%$ of fractures and of these, only four fractures (3.8\%) could not be confirmed. We excluded from all analyses factures not typically associated with osteoporosis, in particular fractures of the face, skull, fingers, and toes. ${ }^{(21,22)}$ We created two categories of fractures: all fractures and minimum trauma fractures. Minimum trauma fractures excluded those that occurred as a result of a fall from a height greater than 6 inches, in a motor vehicle accident, while moving fast (eg, bicycling or skating), while playing sports, or from impact with heavy or fast-moving projectiles.

\section{Measurement of obesity}

At the baseline and each of nine follow-up visits, height and weight were measured using a fixed stadiometer and a digital scale with the participants wearing light clothing and no shoes. The maximum hip circumference was measured over 


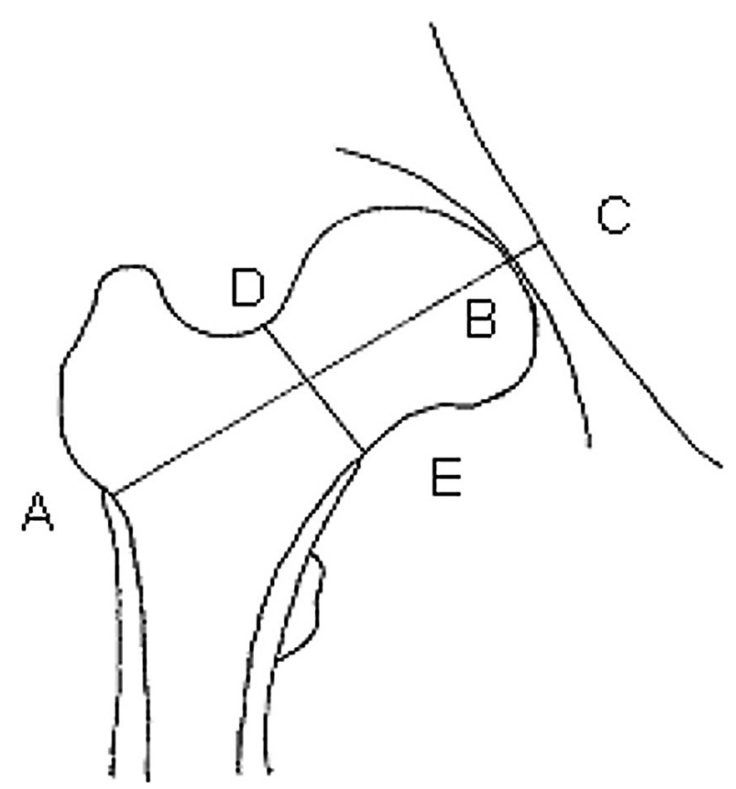

Fig. 1. Femoral neck size measurements and formulae to compute composite femoral neck strength indices. $A B$ is the femoral neck axis length (FNAL), the distance from the base of the greater trochanter to the apex of the femoral head, and DE is the femoral neck width (FNW), the smallest thickness of the femoral neck along any line perpendicular to the femoral neck axis. $C$ is where the femoral neck axis meets the inner pelvic rim. Composite femoral neck strength indices were computed using the following formulae, where BMD refers to the areal (projected 2D) bone mineral density in the femoral neck obtained from DXA:

$$
\begin{aligned}
& \text { Compression Strength Index }(C S I)=\frac{B M D^{*} F N W}{W e i g h t} \\
& \text { Bending Strength Index }(B S I)=\frac{B M D^{*} F W^{2}}{\text { FNAL }^{*} W e i g h t} \\
& \text { Impact Strength Index }(I S I)=\frac{\mathrm{BMD}^{*} F W^{*} F N A L}{H e i g h t^{*} W e i g h t}
\end{aligned}
$$

All three indices were recorded in units of $\mathrm{g} / \mathrm{kg}-\mathrm{m}$. With BMD measured in $\mathrm{g} / \mathrm{cm}^{2}$, FNW and FNAL in $\mathrm{cm}$, weight in $\mathrm{kg}$, and height in meters, CSI and $\mathrm{BSI}$ were scaled by 100 to get values in units of $\mathrm{g} / \mathrm{kg}-\mathrm{m}$.

undergarments (if a participant refused, over light clothing) using a measuring tape with the participants standing with their feet together. Body mass index (BMI) was calculated as weight divided by the square of height $\left(\mathrm{kg} / \mathrm{m}^{2}\right)$. Previous studies suggest nonlinear relationships between $\mathrm{BMI}$ and fracture risk. Therefore, participants were classified into four mutually exclusive BMI categories: low weight $\left(B M I<22 \mathrm{~kg} / \mathrm{m}^{2}\right)$, normal weight $\left(22 \mathrm{~kg} / \mathrm{m}^{2} \leq \mathrm{BMI}<25 \mathrm{~kg} / \mathrm{m}^{2}\right)$, overweight $\left(25 \mathrm{~kg} / \mathrm{m}^{2} \leq\right.$ $\left.\mathrm{BMI}<30 \mathrm{~kg} / \mathrm{m}^{2}\right)$, and obese $\left(30 \mathrm{~kg} / \mathrm{m}^{2} \leq \mathrm{BMI}\right)$. Only 39 women (2.0\%) had $\mathrm{BMI}<18.5 \mathrm{~kg} / \mathrm{m}^{2}$ and therefore women with BMl $<22 \mathrm{~kg} / \mathrm{m}^{2}$, which accounted for $22.5 \%$ of the analytic sample, were categorized as low weight.

\section{Other measurements}

Participants provided the following information at baseline: age (years), race/ethnicity, menopause transition stage (premenopause or early perimenopause: no changes versus some changes in regularity of menses but with no gaps of $\geq 3$ months), physical activity level (summary score combining intensity with frequency of active living, home, and recreational physical activity from modified Baecke interview ${ }^{(23)}$ ), prescription medications used, vitamin $D$ and calcium supplement use, alcohol consumption (abstainer; infrequent: not abstainer, but $\leq 1$ drink per week; light to moderate: $>1$ drink per week, but $\leq 1$ per day; and heavy: $>1$ drink per day), smoking history, and comorbidities. Women who reported use of diabetes medications or had fasting serum glucose $\geq 126 \mathrm{mg} / \mathrm{dL}$ were classified as diabetic. Serum glucose was measured from blood drawn after an overnight fast, using a hexokinase-coupled reaction (Roche Molecular Biochemicals Diagnostics, Indianapolis, IN, USA). Serum CRP level was measured at Medical Research Laboratories (Highland Heights, $\mathrm{KY}, \mathrm{USA})$, using an ultrasensitive rate immunonephelometric method with a lower limit of detection of $0.3 \mathrm{mg} / \mathrm{L}$ (BN100; DadeBehring, Marburg, Germany). The CRP assay within-run coefficient of variation (CV) at CRP concentrations of 0.5 and $22.0 \mathrm{mg} / \mathrm{L}$ were $10 \%-12 \%$ and $5 \%-7 \%$, respectively. During each of the follow-up visits, information on use of medications was collected using interviewer-administered questionnaires.

\section{Statistical analysis}

The first set of analyses was designed to examine the effect of obesity on BMD and bone strength relative to load, and examined cross-sectional associations at the baseline SWAN visit. We first compared the means of the five bone strength estimates (BMD in the lumbar spine and femoral neck, and three composite indices of femoral neck strength relative to load) across BMI categories. Although we had expected to see a J-curve relationship between $\mathrm{BMI}$ and bone strength or fracture hazard, the preliminary analysis found a graded relationship between BMI categories and each of the bone strength estimates and fracture hazard, and we decided to set the low weight group as the reference group. The linear relationships between BMI and bone strength estimates were also tested by entering BMI into the models as a continuous variable rather than a categorical variable.

Multiple linear regression was used to adjust for the following potential confounders, which were also measured at baseline: age (continuous); race/ethnicity; menopause transition stage (premenopause versus early perimenopause); smoking status (never, past, current); alcohol use categories (abstainer, infrequent, light to moderate, heavy); level of physical activity (above median versus below median); current use (yes versus no) of medications from the following four classes (one indicator variable for each): supplementary vitamin $D$, supplementary calcium, other bone-active medications (oral steroids, chemotherapy for breast cancer, aromatase inhibitors, antiepileptics), and central nervous system active medications (tranquilizers, antidepressants, sedatives, sleeping pills); ever/previous use (yes versus no) of oral steroids; ever/previous use of sex steroids (oral estrogen/ progesterone, estrogen patches, birth control pills); history of prior fracture as an adult (after age 20 years); and study site. Use of osteoporosis medications (bisphosphonates, selective estrogen receptor modulators, calcitonin, parathyroid hormone, or vitamin D in pharmacological doses) at baseline was reported by only one participant, and therefore the osteoporosis medications variable was not included in the models. Dunnett's method was used to adjust for multiple comparisons between the low weight (reference category) and the three higher BMI categories. 
To statistically eliminate the (potentially negative) effects of chronic inflammation and diabetes from the association between $\mathrm{BMI}$ and bone strength estimates, we added diabetes status (yes/no) and serum CRP level (which was log-transformed to minimize its skew) to the base models, in a second step.

The second set of analyses examined obesity associations with incidence of fracture over 9 years, and employed Cox proportional hazard regression with time to first fracture as the dependent variable, and baseline BMI as primary predictor. The models were initially adjusted for the same set of baseline covariates as in the cross-sectional base model plus use in at least two consecutive visits during follow up of medications from the following four classes (using one yes/no indicator variable for each class): sex steroid hormones; osteoporosis medications; oral steroids; and other bone-active medications (chemotherapy, aromatase inhibitors, antiepileptics) as timeinvariant covariates. This initial model (the longitudinal base model) estimated the overall effect of obesity on fracture risk. In subsequent models, we added select covariates representing one or more hypothesized obesity-fracture pathways in order to statistically eliminate the effects of the hypothesized pathway(s), and estimate the obesity-fracture association independent of the selected pathway(s). We first added BMD to the model to control for the skeletal loading effect of body weight on BMD. Next, we separately added each composite index of femoral neck strength relative to load to the longitudinal base model to control for the effects of body weight on both bone mass and fall impact forces. We then further adjusted for $\log (\mathrm{CRP})$ and diabetes to remove any residual effects of diabetes and inflammation (which are more prevalent in obese individuals), and thus better isolate the protective effect of soft tissue padding on fracture risk. In the final model, we added a surrogate marker of soft tissue padding around the hip, derived from hip circumference to test if it explained away any remaining protective effect of obesity on fracture risk. The surrogate hip soft tissue measure was created as the residual from race/ethnicity-specific linear regressions of hip circumference on body height.

We conducted all the time-to-event analyses for each of two event types: all fractures and minimum trauma fractures. For analyses of time to first minimum-trauma fractures, follow-up time was censored at the time of the first trauma-associated fracture. $^{(24)}$

A total of 96 (5.0\%) women had one or more covariates missing and the missing values were imputed by single imputation using the expectation maximization (EM) algorithm. ${ }^{(25)}$ All analyses were conducted using SAS version 9.3 (SAS Institute, Inc., Cary, NC, USA). Two-sided $p<0.05$ was considered statistically significant.

\section{Results}

Participants were classified into four BMI categories: 432 (22.5\%) were categorized as low weight (BMI $\left.<22 \mathrm{~kg} / \mathrm{m}^{2}\right), 455(23.6 \%)$ as normal weight $\left(22 \mathrm{~kg} / \mathrm{m}^{2} \leq \mathrm{BMI}<25 \mathrm{~kg} / \mathrm{m}^{2}\right), 469(24.4 \%)$ as overweight $\left(25 \mathrm{~kg} / \mathrm{m}^{2} \leq \mathrm{BMI}<30 \mathrm{~kg} / \mathrm{m}^{2}\right)$, and $568(29.5 \%)$ as obese $\left(30 \mathrm{~kg} / \mathrm{m}^{2} \leq \mathrm{BMI}\right)$. Distributions of characteristics across BMI categories are shown in Table 1. African American women and women in early perimenopause were more likely to be in higher BMI categories. Diabetes, history of previous fracture, less healthier habits (current smoking and less physically active), and use of central nervous system (CNS)-active medications were also more prevalent in higher BMI categories, whereas Chinese and Japanese women and use of supplementary vitamin D and calcium were more common in lower BMl categories.

\section{Cross-sectional associations between BMI and estimates of bone strength}

Multivariable-adjusted means of femoral neck BMD and lumbar spine BMD increased significantly with increasing BMI categories (Table 2, base model). In contrast, the adjusted means of all three composite indices of femoral neck strength relative to load decreased significantly with increasing BMI (all $p<0.001$ ). Consistent with the graded increase in BMD and graded decrease in composite strength indices with increasing BMI categories, BMI as a continuous predictor was also linearly and positively associated with BMD, and linearly and negatively associated with the composite strength indices. Adjusting for diabetes and $\log (\mathrm{CRP})$ only slightly diminished the magnitude of the associations between BMI and the composite indices of strength relative to load (Table 2). Adjusting for diabetes and $\log (\mathrm{CRP})$ had virtually no impact on the associations between $\mathrm{BMI}$ and either lumbar spine BMD or femoral neck BMD.

\section{Association between baseline BMI and incident fracture}

After median follow-up of 9.0 (interquartile range, 8.9-9.1) years, 201 women (10.5\%) had at least one fracture, at a rate of 12.6 per 1000 person-years. Foot (non-toe) and ankle were the most common locations for first incident fracture. In Cox proportional hazard regression, adjusted for age, race/ethnicity, menopause transition stage, smoking status, alcohol use, level of physical activity, use of medications at baseline and during follow-up, history of prior fracture as an adult, and study site, fracture hazard was not significantly associated with BMI (Table 3, base model). After additional adjustment for femoral neck BMD, obesity was significantly associated with increased fracture hazard: relative increment in fracture hazard in obese relative to low weight women: $89 \%$ (95\% confidence intervals $(\mathrm{Cl}), 14 \%$ to $214 \%$ ) (Table 3, model 2a). The relative increment in fracture hazard in obese women compared to normal weight women was also statistically significant: $78 \%(95 \% \mathrm{Cl}, 13 \%$ to $181 \%, p=0.01)$. In stark contrast, obesity was significantly associated with decreased fracture hazard when adjusted instead for any of the composite indices of femoral neck strength relative to load: relative decrement in fracture hazard in obese relative to low weight women was $57 \%$ ( $95 \% \mathrm{Cl}, 24 \%$ to $76 \%)$ after adjusting for CSI, $41 \%(95 \% \mathrm{Cl}, 1 \%$ to $65 \%)$ after adjusting for $\mathrm{BSI}$, and $53 \%$ (95\% CI, $16 \%$ to $74 \%$ ) after adjusting for ISI (Table 3, model 3). The relative decrement in fracture hazard in obese women relative to normal weight women after adjusting for CSI was 39\% $(p=0.053)$. Addition of diabetes and $\log (\mathrm{CRP})$ to the model with CSI minimally affected the associations between BMI and fracture hazard (Table 3, model 4). Further adjustment for hip soft tissue attenuated the associations between BMI and fracture hazard and made them statistically nonsignificant (Table 3, model 5). Similar results were observed when diabetes, log(CRP), and hip soft tissue were added to Models $3 \mathrm{~b}$ and $3 \mathrm{c}$ (the models with BSI and ISI)—data not shown.

Consistent with the graded associations between BMI categories and fracture hazard in models 2 through 4 (Table 3), BMI as a continuous predictor was linearly and positively associated with fracture hazard after adjusting for femoral neck or lumbar spine BMD (Table 3, models $2 a$ and $2 b$ ), but linearly and negatively associated with fracture hazard after adjusting for composite 
Table 1. Participant Characteristics at the Baseline Visit in the Complete Study Sample and by Body Mass Index Categories ${ }^{a}$

\begin{tabular}{|c|c|c|c|c|c|c|}
\hline Characteristics & $\begin{array}{c}\text { All } \\
(n=1924)\end{array}$ & $\begin{array}{l}\text { Low weight }^{\mathrm{b}} \\
\quad(n=432)\end{array}$ & $\begin{array}{l}\text { Normal }^{\mathrm{b}} \\
(n=455)\end{array}$ & $\begin{array}{l}\text { Overweight }^{\mathrm{b}} \\
\quad(n=469)\end{array}$ & $\begin{array}{l}\text { Obese }^{b} \\
(n=568)\end{array}$ & $\begin{array}{l}p \text { for } \\
\text { trend }^{c}\end{array}$ \\
\hline Age (years) & $45.9(2.7)$ & $45.7(2.7)$ & $45.9(2.7)$ & $46.1(2.7)$ & $45.8(2.7)$ & 0.47 \\
\hline Height (cm) & $162.3(6.5)$ & $161.0(6.8)$ & $161.8(6.5)$ & $163.2(6.4)$ & $162.8(6.2)$ & $<0.001$ \\
\hline Weight (kg) & $72.6(19.3)$ & $52.7(5.4)$ & $61.6(5.5)$ & $72.9(6.9)$ & $96.3(14.6)$ & $<0.001$ \\
\hline BMI $\left(k g / m^{2}\right)$ & $27.5(6.9)$ & $20.3(1.3)$ & $23.5(0.9)$ & $27.3(1.4)$ & $36.3(5.1)$ & $<0.001$ \\
\hline Hip circumference $(\mathrm{cm})$ & $105.3(13.9)$ & $91.2(4.3)$ & $97.7(4.5)$ & $105.5(5.4)$ & $122.0(11.3)$ & $<.0001$ \\
\hline Waist circumference $(\mathrm{cm})$ & $84.6(15.2)$ & $68.9(4.0)$ & $76.0(4.4)$ & $85.3(6.7)$ & $103.1(11.3)$ & $<0.001$ \\
\hline Race/ethnicity, $n$ (\%) & & & & & & $<0.001$ \\
\hline White & $963(50.1)$ & $185(42.8)$ & $236(51.9)$ & $248(52.9)$ & $294(51.8)$ & \\
\hline African American & $503(26.1)$ & $38(8.8)$ & $69(15.2)$ & $141(30.1)$ & $255(44.9)$ & \\
\hline Chinese & $220(11.4)$ & $100(23.2)$ & $72(15.8)$ & $39(8.3)$ & $9(1.6)$ & \\
\hline Japanese & $238(12.4)$ & $109(25.2)$ & $78(17.1)$ & $41(8.7)$ & $10(1.8)$ & \\
\hline Menopause transition stage, $n(\%)^{d}$ & & & & & & 0.009 \\
\hline Premenopausal & $1087(56.5)$ & $260(60.2)$ & $266(58.5)$ & $264(56.3)$ & $297(52.3)$ & \\
\hline Early perimenopausal & 837 (43.5) & $172(39.8)$ & $189(41.5)$ & $205(43.7)$ & $271(47.7)$ & \\
\hline Smoking Status, $n$ (\%) & & & & & & $<0.001$ \\
\hline Never smoked & $1142(59.8)$ & $280(65.1)$ & $287(63.2)$ & $266(57.2)$ & $309(55.0)$ & \\
\hline Ex-smoker & $486(25.4)$ & $92(21.4)$ & $112(24.7)$ & $128(27.5)$ & $154(27.4)$ & \\
\hline Current smoker & $283(14.8)$ & $58(13.5)$ & $55(12.1)$ & $71(15.3)$ & $99(17.6)$ & \\
\hline Alcohol consumption, $n(\%)^{\mathrm{e}}$ & & & & & & $<0.001$ \\
\hline Abstainer & $992(51.6)$ & $199(46.3)$ & $218(48.0)$ & $235(50.1)$ & $340(59.9)$ & \\
\hline Infrequent & $179(9.3)$ & 47 (10.9) & $37(8.2)$ & $41(8.7)$ & $54(9.5)$ & \\
\hline Light to moderate & $489(25.5)$ & $112(26.1)$ & $125(27.5)$ & $121(25.8)$ & $131(23.1)$ & \\
\hline Heavy & $261(13.6)$ & $72(16.7)$ & $74(16.3)$ & $72(15.4)$ & $43(7.6)$ & \\
\hline Physical activity level, $n$ (\%) & & & & & & $<0.001$ \\
\hline Above median & $945(50.6)$ & $247(59.1)$ & $257(57.8)$ & $243(53.3)$ & $198(36.2)$ & \\
\hline Below median & $921(49.4)$ & $171(40.9)$ & $188(42.3)$ & $213(46.7)$ & $349(63.8)$ & \\
\hline History of prior fracture as adult, $n(\%)$ & $353(18.4)$ & $57(13.2)$ & $72(15.8)$ & $101(21.5)$ & $123(21.7)$ & $<0.001$ \\
\hline \multicolumn{7}{|l|}{ Current use of medications, $n(\%)$} \\
\hline Supplementary vitamin D & $742(38.6)$ & $177(41.2)$ & $175(38.6)$ & $182(38.8)$ & $208(36.6)$ & 0.17 \\
\hline Supplementary calcium & $863(44.9)$ & $218(50.7)$ & $212(46.7)$ & $208(44.4)$ & $225(39.6)$ & $<0.001$ \\
\hline CNS active medications ${ }^{f}$ & 199 (10.3) & $39(9.0)$ & $37(8.1)$ & $47(10.0)$ & $76(13.4)$ & 0.01 \\
\hline Other bone-active medications ${ }^{\mathrm{g}}$ & $44(2.3)$ & 8 (1.9) & $7(1.5)$ & $13(2.8)$ & $16(2.8)$ & 0.17 \\
\hline \multicolumn{7}{|l|}{ Previous use of medications, $n(\%)$} \\
\hline Sex steroid hormones & $1419(73.8)$ & $287(66.4)$ & $330(72.5)$ & $357(76.1)$ & $445(78.4)$ & $<0.001$ \\
\hline Oral steroids & $92(4.8)$ & $13(3.0)$ & $20(4.4)$ & $21(4.5)$ & $38(6.7)$ & 0.008 \\
\hline Diabetes mellitus, $n(\%)^{\mathrm{h}}$ & $88(4.6)$ & $3(0.7)$ & $7(1.5)$ & $10(2.1)$ & $68(12.0)$ & $<0.001$ \\
\hline FNAL $(\mathrm{cm})$ & $8.97(0.51)$ & $8.98(0.54)$ & $8.98(0.51)$ & $9.01(0.51)$ & $8.91(0.48)$ & 0.09 \\
\hline FNW $(\mathrm{cm})$ & $2.75(0.20)$ & $2.73(0.20)$ & $2.73(0.20)$ & $2.75(0.20)$ & $2.78(0.20)$ & $<0.001$ \\
\hline Lumbar spine BMD (g/cm²) & $1.07(0.13)$ & $1.00(0.11)$ & $1.04(0.12)$ & $1.09(0.13)$ & $1.13(0.13)$ & $<0.001$ \\
\hline Femoral neck BMD $\left(\mathrm{g} / \mathrm{cm}^{2}\right)$ & $0.84(0.13)$ & $0.75(0.10)$ & $0.80(0.10)$ & $0.85(0.11)$ & $0.95(0.13)$ & $<0.001$ \\
\hline $\mathrm{CSI}(\mathrm{g} / \mathrm{kg}-\mathrm{m})$ & $3.31(0.64)$ & $3.90(0.55)$ & $3.54(0.47)$ & $3.20(0.43)$ & $2.76(0.45)$ & $<0.001$ \\
\hline BSI (g/kg-m) & $1.02(0.22)$ & $1.19(0.21)$ & $1.08(0.19)$ & $0.98(0.16)$ & $0.87(0.16)$ & $<0.001$ \\
\hline ISI (g/kg-m) & $0.18(0.04)$ & $0.22(0.03)$ & $0.20(0.03)$ & $0.18(0.02)$ & $0.15(0.03)$ & $<0.001$ \\
\hline
\end{tabular}

Abbreviations: BMI, body mass index; CNS, central nervous system; FNAL, femoral neck axis length; FNW, femoral neck width; CSI, compression strength index; $\mathrm{BSI}$, bending strength index; ISI, impact strength index.

${ }^{\mathrm{a}}$ Mean and standard deviation shown for continuous variables and number of participants and percentage shown for categorical variables.

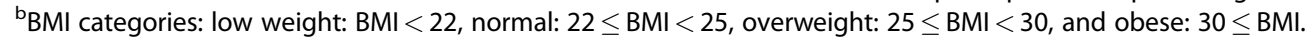

${ }^{c} p$ value for trend across increasing BMI categories was calculated using the Jonckheere-Terpstra test.

${ }^{d}$ Women were classified as premenopausal if they had experienced at least one menstrual period in the last 3 months with no change in the regularity of their menstrual bleeding during the last year and early perimenopausal if they had experienced at least one menstrual period in the last 3 months with some change in the regularity of their menstrual bleeding during the last year.

eWomen were classified as abstainer if they consumed no alcohol, infrequent if they consumed less than one drink per week, light to moderate if they consumed more than one drink per week but less than one drink per day, and heavy if they consumed more than one drink per day.

${ }^{f}$ CNS active medications include tranquilizers, antidepressants, sedatives, and sleeping pills.

${ }^{9}$ Other bone-active medications include oral steroids, chemotherapy for breast cancer, aromatase inhibitors, and antiepileptics.

hWomen who reported use of diabetes medications or had fasting serum glucose $\geq 126 \mathrm{mg} / \mathrm{dL}$ were classified as diabetic. 


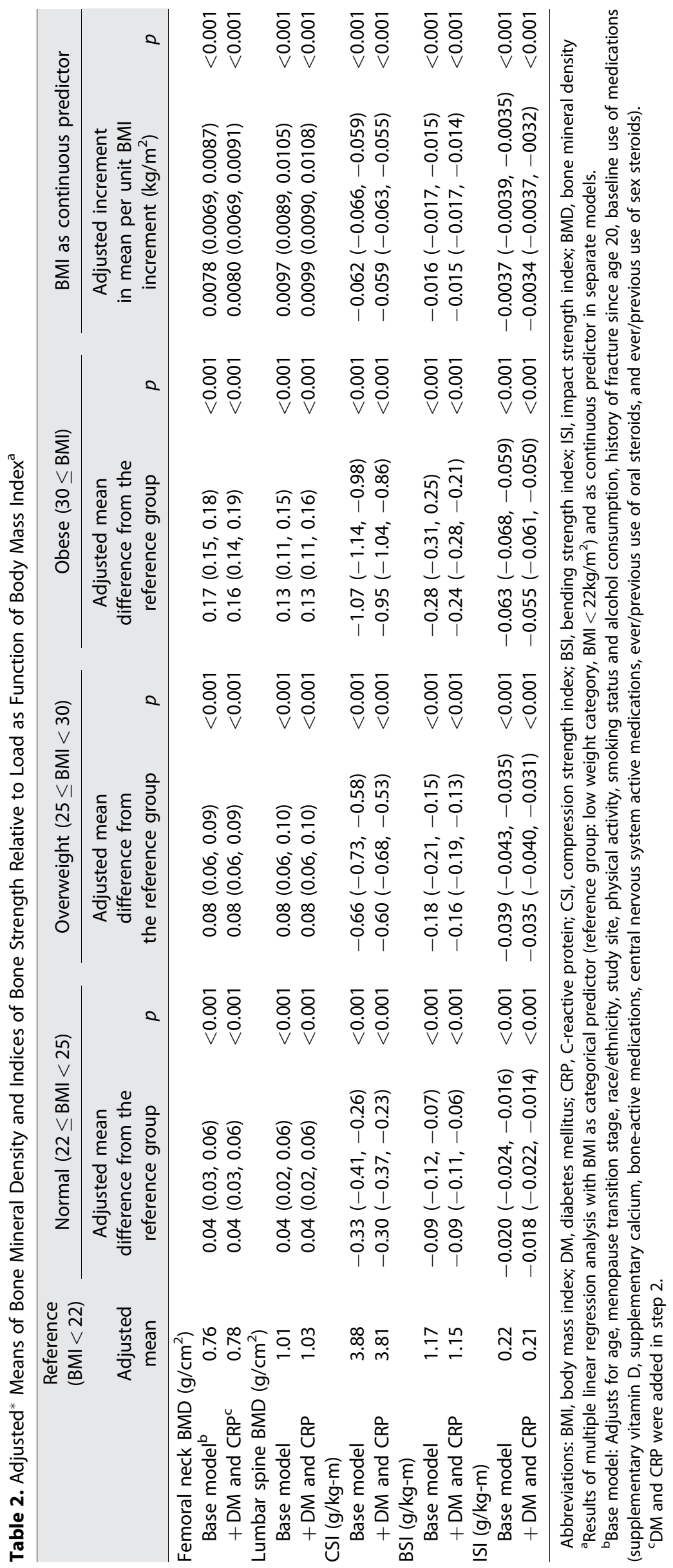


Table 3. Adjusted* Associations Between Body Mass Index and Incident Fracture Before and After Controlling for Different Pathways ${ }^{\mathrm{a}}$

\begin{tabular}{|c|c|c|c|c|c|c|c|c|}
\hline & \multirow{2}{*}{\multicolumn{2}{|c|}{$\begin{array}{r}\text { Normal }(22 \leq \mathrm{BMI}<25) \\
\text { Ref: Low weight }\end{array}$}} & \multirow{2}{*}{\multicolumn{2}{|c|}{$\begin{array}{c}\text { Overweight }(25 \leq \mathrm{BMI}<30) \\
\text { Ref: Low weight }\end{array}$}} & \multirow{2}{*}{\multicolumn{2}{|c|}{$\begin{array}{l}\text { Obese }(30 \leq \mathrm{BMI}) \\
\text { Ref: Low weight }\end{array}$}} & \multirow{2}{*}{\multicolumn{2}{|c|}{$\begin{array}{l}\text { BMI as continuous predictor } \\
\text { Per unit BMI increment }\left(\mathrm{kg} / \mathrm{m}^{2}\right)\end{array}$}} \\
\hline & & & & & & & & \\
\hline & $\mathrm{HR}(95 \% \mathrm{Cl})$ & $p$ & $\mathrm{HR}(95 \% \mathrm{Cl})$ & $p$ & $\mathrm{HR}(95 \% \mathrm{Cl})$ & $p$ & $\mathrm{HR}(95 \% \mathrm{Cl})$ & $p$ \\
\hline 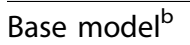 & $0.91(0.58,1.41)$ & 0.67 & $1.02(0.66,1.59)$ & 0.93 & $1.01(0.64,1.59)$ & 0.97 & $1.01(0.98,1.03)$ & 0.58 \\
\hline Model 2a & $1.06(0.68,1.67)$ & 0.79 & $1.32(0.84,2.08)$ & 0.22 & $1.89(1.14,3.14)$ & 0.01 & $1.05(1.02,1.07)$ & $<.001$ \\
\hline Model 2b & $1.03(0.66,1.62)$ & 0.89 & $1.27(0.81,1.99)$ & 0.30 & $1.50(0.93,2.42)$ & 0.10 & $1.03(1.005,1.06)$ & 0.02 \\
\hline Model 3a & $0.70(0.44,1.10)$ & 0.12 & $0.59(0.36,0.97)$ & 0.04 & $0.43(0.24,0.76)$ & 0.004 & $0.96(0.93,0.99)$ & 0.006 \\
\hline Model 3b & $0.76(0.48,1.20)$ & 0.24 & $0.72(0.45,1.16)$ & 0.18 & $0.59(0.35,0.99)$ & 0.048 & $0.98(0.95,1.003)$ & 0.08 \\
\hline Model 3c & $0.71(0.45,1.13)$ & 0.15 & $0.63(0.38,1.03)$ & 0.07 & $0.47(0.26,0.84)$ & 0.01 & $0.96(0.93,0.99)$ & 0.02 \\
\hline Model 4 & $0.71(0.45,1.13)$ & 0.15 & $0.62(0.37,1.02)$ & 0.06 & $0.43(0.23,0.81)$ & 0.009 & $0.95(0.92,0.99)$ & 0.005 \\
\hline Model 5 & $0.76(0.48,1.22)$ & 0.26 & $0.72(0.42,1.23)$ & 0.23 & $0.65(0.31,1.40)$ & 0.27 & $0.98(0.91,1.04)$ & 0.47 \\
\hline
\end{tabular}

\footnotetext{
Abbreviations: $\mathrm{BMI}$, body mass index; $\mathrm{HR}$, hazard ratio, $\mathrm{Cl}$, confidence intervals.

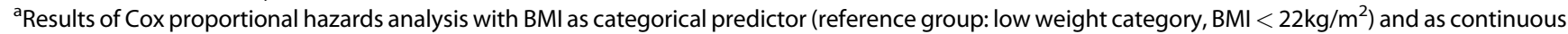
predictor in separate models.

${ }^{\mathrm{b}}$ Base model: age, menopause transition stage, race/ethnicity, study site, physical activity, smoking status and alcohol consumption, history of fracture since age 20, baseline use of medications (supplementary vitamin D, supplementary calcium, bone-active medications, central nervous system active medications, ever/previous use of oral steroids, and ever/previous use of sex steroids) and use of medications during the follow-up (sex steroid hormones, osteoporosis medications, oral steroids, and other bone-active medications).

Model 2a: adjusted for covariates in base model plus femoral neck BMD. Model 2b: adjusted for covariates in base model plus lumbar spine BMD. Model 3a: adjusted for covariates in base model plus compression strength index. Model $3 \mathrm{~b}$ : adjusted for covariates in base model plus bending strength index. Model 3c: adjusted for covariates in base model plus impact strength index.

Model 4: adjusted for covariates in model 3a plus diabetes status and log (C-reactive protein). Model 5: adjusted for covariates in model 4 plus a surrogate measure of hip soft tissue.
}

indices of femoral neck strength relative to load (Table 3, models $3 a$ and $3 c$ ). Similarly, the linear and negative relationship between BMI (as a continuous predictor) and fracture hazard became nonsignificant when hip soft tissue was added to the model (Table 3, model 5).

\section{Association between baseline BMI and incident minimum} trauma fracture

After the 9-year follow-up, 82 women (4.3\%) had at least one minimum trauma fracture, at a rate of 5.1 per 1000 person-years. Findings for minimum trauma fracture were similar to the ones for all fracture with respect to the direction and magnitude of the associations with BMI (Table 4). However, reflecting the smaller number of events, fewer associations reached levels of statistical significance.

\section{Discussion}

This study was designed to test multiple hypothesized pathways by which obesity might affect fracture risk: (1) increased BMD in response to greater skeletal loading, (2) increased impact forces in a fall (load), (3) deleterious effects of chronic inflammation and diabetes (common in obesity) on bone mass, and (4) absorption of impact forces by soft tissue padding. Using data from a multiethnic cohort of 1924 women going through the menopause transition, we demonstrated that although higher BMI was indeed associated with higher BMD (consistent with previous studies ${ }^{(2,3)}$, it is in fact associated with lower indices of bone strength relative to load. These associations between BMI and the bone strength estimates were monotonic. These findings suggest that although obesity may increase BMD, the increase in bone strength is not commensurate with the increase in fall impact forces. Further adjustment for the detrimental effects of diabetes and inflammation did not substantially alter the associations between high BMI and low bone strength.
We also demonstrated that adjusted for BMD, obesity was associated with increased fracture hazard. Increased fracture hazard in obesity, when adjusted for BMD, has been seen in previous studies $^{(10,26)}$ and is consistent with the hypothesis that larger fall impact forces in obesity increase fracture risk. We also demonstrated that obesity was associated with decreased fracture hazard after adjustment for any of the three composite indices of bone strength relative to fall impact forces. Since controlling for the composite indices statistically eliminates the effects of obesity on both bone strength and fall impact forces without eliminating the effect of soft tissue padding, this is consistent with a fracture protection effect of soft tissue padding. Such a protective role is further supported by the observation that additional adjustment for a surrogate marker for hip soft tissue padding attenuated the remaining obesity-fracture association and made it statistically nonsignificant.

Taken together, these findings provide empirical evidence for the hypothesized pleiotropic effects of obesity on fracture risk. First, bone mass increases in response to the excess weight. Second, obesity increases fracture risk by increasing impact forces in a fall. The increase in bone mass in obesity is not commensurate with the increased fall impact forces. Third, the reduction in bone strength relative to load is to some degree offset by the protection conferred by increased soft tissue padding. The balance between these factors determines the overall fracture risk in an individual.

Because the magnitude of these competing effects can vary from body site to body site, the relationship between obesity and fracture will be both site-specific and person-specific. For instance, the adaptive increase in BMD due to greater skeletal loading will be more pronounced at weight-bearing sites such as the hip and spine than in the forearm and wrist. Similarly, the protection conferred by soft tissue mass will be greatest at body sites where it is most abundant, and the localization of soft tissue can vary from person to person. For instance, gynecoid obesity, where most fat accumulation is around the hip, should provide 
Table 4. Adjusted* Associations Between Body Mass Index and Incident Minimum Trauma Fracture Before and After Controlling for Different Pathways ${ }^{a}$

\begin{tabular}{|c|c|c|c|c|}
\hline & \multirow{2}{*}{\multicolumn{2}{|c|}{$\begin{array}{c}\begin{array}{c}\text { Obese } \\
(30 \leq \mathrm{BMI})\end{array} \\
\text { Ref: Low } \\
\text { weight }\end{array}$}} & \multirow{2}{*}{\multicolumn{2}{|c|}{$\begin{array}{l}\text { BMI as continuous } \\
\text { predictor } \\
\text { Per unit } \mathrm{BMI} \\
\text { increment }\left(\mathrm{kg} / \mathrm{m}^{2}\right)\end{array}$}} \\
\hline & & & & \\
\hline & HR (95\% Cl) & $p$ & $\mathrm{HR}(95 \% \mathrm{Cl})$ & $p$ \\
\hline Base model $^{b}$ & $1.06(0.49,2.28)$ & 0.88 & $1.02(0.98,1.05)$ & 0.35 \\
\hline Model 2a & $1.78(0.77,4.13)$ & 0.18 & $1.05(1.01,1.09)$ & 0.01 \\
\hline Model 2b & $1.58(0.71,3.51)$ & 0.26 & $1.04(1.004,1.08)$ & 0.03 \\
\hline Model 3a & $0.33(0.13,0.87)$ & 0.02 & $0.96(0.92,1.007)$ & 0.10 \\
\hline Model 3b & $0.47(0.20,1.10)$ & 0.08 & $0.98(0.94,1.02)$ & 0.23 \\
\hline Model 3c & $0.39(0.15,1.005)$ & 0.051 & $0.97(0.93,1.02)$ & 0.22 \\
\hline Model 4 & $0.29(0.10,0.80)$ & 0.02 & $0.95(0.90,1.001)$ & 0.053 \\
\hline Model 5 & $0.30(0.09,1.03)$ & 0.055 & $0.95(0.86,1.05)$ & 0.31 \\
\hline
\end{tabular}

Abbreviations: $\mathrm{BMI}$, body mass index; $\mathrm{HR}$, hazard ratio, $\mathrm{Cl}$, confidence intervals.

${ }^{\mathrm{a}}$ Results of Cox proportional hazards analysis with BMI as categorical predictor (reference group: low weight category, $\mathrm{BMI}<22 \mathrm{~kg} / \mathrm{m}^{2}$ ) and as continuous predictor in separate models.

${ }^{b}$ Base model: age, menopause transition stage, race/ethnicity, study site, physical activity, smoking status and alcohol consumption, history of fracture since age 20, baseline use of medications (supplementary vitamin $D$, supplementary calcium, bone-active medications, central nervous system active medications, ever/previous use of oral steroids, and ever/previous use of sex steroids) and use of medications during the follow-up (sex steroid hormones, osteoporosis medications, oral steroids, and other bone-active medications). Model 2a: adjusted for covariates in base model plus femoral neck BMD. Model $2 \mathrm{~b}$ : adjusted for covariates in base model plus lumbar spine BMD. Model 3a: adjusted for covariates in base model plus compression strength index. Model 3b: adjusted for covariates in base model plus bending strength index. Model 3c: adjusted for covariates in base model plus impact strength index. Model 4: adjusted for covariates in model 3a plus diabetes status and $\log$ (C-reactive protein). Model 5: adjusted for covariates in model 4 plus a surrogate measure of hip soft tissue.

protection against hip fractures, but not necessarily protect against fractures at other appendicular sites. This is consistent with previous studies which found lower hip fracture risk ${ }^{(4,11,12,14)}$ but higher risk of fractures elsewhere ${ }^{(11-15)}$ in obese compared to non-obese women.

Previous studies have found that both inflammation and diabetes, which are more prevalent in obesity, have deleterious effects on bone strength and fracture risk ${ }^{(6-9)}$ and that diabetes is also associated with increased fall risk. ${ }^{(27,28)}$ The observed negative associations of obesity with both bone strength relative to load and fracture risk independent of inflammation and diabetes in this current study suggests that there are other direct mechanisms by which obesity is deleterious to bone health. Adipose tissue is considered an endocrine organ producing adipokines such as adiponectin and leptin, which have been implicated in bone metabolism. ${ }^{(29)}$ Increased bone marrow fat in obesity may also have deleterious effects on bone. ${ }^{(30)}$ Other possible mechanisms include osteoarthritis (which increases fall risk), ${ }^{(11,31)}$ prediabetes, hyperlipidemia, hypertension, and vascular atherosclerosis (which may reduce blood and oxygen supply to bone), all of which are more prevalent in obese individuals.

It should be noted that the composite strength indices employed in this study are structural measures based on macroscopic measurements from DXA scans and body size derived from structural engineering principles. The indices ignore important microscopic features such as differences in microarchitecture, and a recent case control study did demonstrate that obesity-induced improvements in microarchitecture may also not be commensurate with the increased body weight. ${ }^{(32)}$ In addition, the strength indices were derived using the simplifying assumption that the femoral neck is a simple cylinder and do not take into account other aspects of shape such as femoral neck-shaft angle, which may also be influenced by body composition ${ }^{(4,33)}$ and associated with hip fracture risk. ${ }^{(34)}$ These other pathways from obesity to fracture risk need further investigation.

Our study has other limitations to be noted. First, fractures were self-reported, but fractures after visit 6 were confirmed by medical records review. It is still possible that we overlooked clinically silent vertebral fractures. Also, the exact date of fracture was not recorded until the medical records review process began; thus, fractures that occurred prior to that time were assumed to occur at the midpoint between the current and prior visit. This could bias our estimates. ${ }^{(35)}$ Second, we employed BMI as a measure of obesity and did not assess the role of truncal versus appendicular distribution of fat or distinguish fat mass from lean mass. We also did not differentiate between visceral and subcutaneous adipose tissues, each of which may have different biological implications. ${ }^{(36)}$ Third, the composite strength indices were designed to quantify bone strength in the femoral neck, but fractures in the femoral neck were relatively rare in this cohort of middle-aged women (less than $5 \%$ of all fractures). However, just like low or high BMD in the femoral neck is a reflection of low or high bone mass more generally, ${ }^{(22)}$ femoral neck strength indices also reflect bone strength elsewhere in the individual and predict fracture risk more generally. ${ }^{(20,37)}$ Fourth, the incidence of fracture was low in this cohort of middle-aged women, precluding analyses by specific locations of bone fractures and limiting our power to detect residual BMI effects after adjusting for hip soft tissue. Lastly, we did not take into account the change in BMD or bone strength indices during follow-up, which may also be influenced by obesity. Further studies are warranted to assess the generalizability of our findings to other populations, such as men, older postmenopausal women, and younger premenopausal women.

In summary, this study provided empirical evidence for at least three major mechanisms by which obesity may influence fracture risk: adaptive increase in BMD, increased impact forces in a fall, and soft tissue padding to absorb impact forces. Our findings suggest that the assessment of fracture risk has to go beyond measurement of BMD to include assessment of bone strength relative to load and the extent of soft tissue over potential impact sites.

\section{Disclosures}

All authors state that they have no conflicts of interest.

\section{Acknowledgments}

The Study of Women's Health Across the Nation (SWAN) has grant support from the National Institutes of Health (NIH), DHHS, through the National Institute on Aging (NIA), the National Institute of Nursing Research (NINR), and the NIH Office of Research on Women's Health (ORWH) (grants U01NR004061, U01AG012505, 
U01AG012535, U01AG012531, U01AG012539, U01AG012546, U01AG012553, U01AG012554, and U01AG012495). The Hip Strength Through the Menopausal Transition Study is supported by the NIA (AG026463). The content of this manuscript is solely the responsibility of the authors and does not necessarily represent the official views of the NIA, NINR, ORWH, or the NIH.

Clinical Centers: University of Michigan, Ann Arbor-Siobán Harlow, PI 2011-present, MaryFran Sowers, PI 1994-2011; Massachusetts General Hospital, Boston, MA-Joel Finkelstein, PI 1999-present, Robert Neer, PI 1994-1999; Rush University, Rush University Medical Center, Chicago, IL-Howard Kravitz, PI 2009-present, Lynda Powell, PI 1994-2009; University of California, Davis/Kaiser-Ellen Gold, PI; University of California, Los Angeles-Gail Greendale, Pl; Albert Einstein College of Medicine, Bronx, NY-Carol Derby, PI 2011-present, Rachel Wildman, PI 2010-2011, Nanette Santoro, PI 2004-2010; University of Medicine and Dentistry-New Jersey Medical School, Newark, NJ-Gerson Weiss, PI 1994-2004; and the University of Pittsburgh, Pittsburgh, PA-Karen Matthews, PI.

NIH Program Office: National Institute on Aging, Bethesda, MD-Winifred Rossi 2012-present, Sherry Sherman 1994-2012, Marcia Ory 1994-2001; National Institute of Nursing Research, Bethesda, MD-Program Officers.

Central Laboratory: University of Michigan, Ann Arbor-Daniel McConnell (Central Ligand Assay Satellite Services).

Coordinating Center: University of Pittsburgh, Pittsburgh, PA-Maria Mori Brooks, PI 2012-present, Kim Sutton-Tyrrell, PI 2001-2012; New England Research Institutes, Watertown, MASonja McKinlay, PI 1995-2001.Steering Committee: Susan Johnson, Current Chair; Chris Gallagher, Former Chair.

We thank the study staff at each site and all the women who participated in SWAN.

Authors' roles Study Concept: AK, SI, GG, and JC. Data analysis: SI. Obtain funding: AK, GG, and JC. Data interpretation: SI, AK, GG, and CC. Drafting manuscript: SI. Revising manuscript content: SI, AK GG, JC, CN, CKG, and KR. Approving final version of manuscript: SI, AK GG, JC, CN, CKG, and KR. SI takes responsibility for the integrity of the data analysis.

\section{References}

1. De Laet $C$, Kanis JA, Oden A, Johanson $H$, Johnell $O$, Delmas $P$, et al. Body mass index as a predictor of fracture risk: a meta-analysis. Osteoporos Int. 2005;16:1330-8.

2. Looker AC, Flegal KM, Melton LJ 3rd. Impact of increased overweight on the projected prevalence of osteoporosis in older women. Osteoporos Int. 2007;18:307-13.

3. Felson DT, Zhang Y, Hannan MT, Anderson JJ. Effects of weight and body mass index on bone mineral density in men and women: the Framingham study. J Bone Miner Res. 1993;8:567-73.

4. Beck TJ, Petit MA, Wu G, LeBoff MS, Cauley JA, Chen Z. Does obesity really make the femur stronger? BMD, geometry, and fracture incidence in the women's health initiative-observational study. J Bone Miner Res. 2009;24:1369-79.

5. Beck TJ, Oreskovic TL, Stone KL, Ruff CB, Ensrud K, Nevitt MC, et al. Structural adaptation to changing skeletal load in the progression toward hip fragility: the study of osteoporotic fractures. J Bone Miner Res. 2001;16:1108-19.

6. Ishii S, Cauley JA, Greendale GA, Crandall CJ, Danielson ME, Ouchi Y, et al. C-reactive protein, bone strength, and 9-year fracture risk: Data from the study of women's health across the nation (SWAN). J Bone Miner Res. 2013;28:1688-98.

7. Ishii S, Cauley JA, Crandall CJ, Srikanthan P, Greendale GA, Huang MH, et al. Diabetes and femoral neck strength: findings from the Hip
Strength Across the Menopausal Transition Study. J Clin Endocrinol Metab. 2012;97:190-7.

8. Cauley JA, Danielson ME, Boudreau RM, Forrest KY, Zmuda JM, Pahor $M$, et al. Inflammatory markers and incident fracture risk in older men and women: the Health Aging and Body Composition Study. J Bone Miner Res. 2007;22:1088-95.

9. Vestergaard P. Discrepancies in bone mineral density and fracture risk in patients with type 1 and type 2 diabetes-a meta-analysis. Osteoporos Int. 2007;18:427-44.

10. Nielson CM, Marshall LM, Adams AL, LeBlanc ES, Cawthon PM, Ensrud $\mathrm{K}$, et al. BMI and fracture risk in older men: the osteoporotic fractures in men study (MrOS). J Bone Miner Res. 2011;26:496-502.

11. Compston JE, Watts NB, Chapurlat R, Cooper C, Boonen S, Greenspan $\mathrm{S}$, et al. Obesity is not protective against fracture in postmenopausal women: GLOW. Am J Med. 2011;124:1043-50.

12. Prieto-Alhambra $D$, Premaor MO, Fina Aviles F, Hermosilla $E$, Martinez-Laguna D, Carbonell-Abella C, et al. The association between fracture and obesity is site-dependent: a population-based study in postmenopausal women. J Bone Miner Res. 2012;27:294300.

13. Compston JE, Flahive J, Hosmer DW, Watts NB, Siris ES, Silverman S, et al. Relationship of weight, height, and body mass index with fracture risk at different sites in postmenopausal women: The global longitudinal study of osteoporosis in women (GLOW). J Bone Miner Res. 2014;29:487-93.

14. Premaor MO, Compston JE, Fina Aviles F, Pages-Castella A, Nogues $X$, Diez-Perez $A$, et al. The association between fracture site and obesity in men: A population-based cohort study. J Bone Miner Res. 2013;28:1771-7.

15. Tanaka S, Kuroda T, Saito M, Shiraki M. Overweight/obesity and underweight are both risk factors for osteoporotic fractures at different sites in Japanese postmenopausal women. Osteoporos Int. 2013;24:69-76.

16. Matthews KA, Crawford SL, Chae CU, Everson-Rose SA, Sowers MF, Sternfeld B, et al. Are changes in cardiovascular disease risk factors in midlife women due to chronological aging or to the menopausal transition? J Am Coll Cardiol. 2009;54:2366-73.

17. Randolph JF Jr, Zheng H, Sowers M, Crandall C, Crawford S, Gold EB, et al. Change in follicle-stimulating hormone and estradiol across the menopausal transition: effect of age at the final menstrual period. J Clin Endocrinol Metab. 2011;96:746-54.

18. Sowers M, Crawford S, Sternfeld B, Morganstein D, Gold E, Greendale $\mathrm{G}$, et al. Design, survey, sampling and recruitment methods of SWAN: a multi-center, multi-ethnic, community based cohort study of women and the menopausal transition. In: Lobo RA, Kelsey J, Marcus M, editors. Menopause: biology and pathobiology. San Diego: Academic Press; 2000. pp175-88.

19. Karlamangla AS, Barrett-Connor E, Young J, Greendale GA. Hip fracture risk assessment using composite indices of femoral neck strength: the Rancho Bernardo study. Osteoporos Int. 2004;15: 62-70.

20. Ishii S, Greendale GA, Cauley JA, Crandall CJ, Huang MH, Danielson $\mathrm{ME}$, et al. Fracture risk assessment without race/ethnicity information. J Clin Endocrinol Metab. 2012;97:3593-602.

21. Seeley DG, Browner WS, Nevitt MC, Genant HK, Scott JC, Cummings $\mathrm{SR}$. Which fractures are associated with low appendicular bone mass in elderly women? The Study of Osteoporotic Fractures Research Group. Ann Intern Med. 1991;115:837-42.

22. Stone KL, Seeley DG, Lui LY, Cauley JA, Ensrud K, Browner WS, et al. $B M D$ at multiple sites and risk of fracture of multiple types: long-term results from the Study of Osteoporotic Fractures. J Bone Miner Res. 2003;18:1947-54.

23. Baecke JA, Burema J, Frijters JE. A short questionnaire for the measurement of habitual physical activity in epidemiological studies. Am J Clin Nutr. 1982;36:936-42.

24. Prentice RL, Kalbfleisch JD, Peterson AV Jr, Flournoy N, Farewell VT, Breslow NE. The analysis of failure times in the presence of competing risks. Biometrics. 1978;34:541-54.

25. Graham JW. Missing data analysis: making it work in the real world. Annu Rev Psychol. 2009;60:549-76. 
26. Johansson H, Kanis JA, Oden A, McCloskey E, Chapurlat RD, Christiansen $C$, et al. A meta-analysis of the association of fracture risk and body mass index in women. J Bone Miner Res. 2014;29:223-33.

27. Schwartz AV, Hillier TA, Sellmeyer DE, Resnick HE, Gregg E, Ensrud KE, et al. Older women with diabetes have a higher risk of falls: a prospective study. Diabetes Care. 2002;25:1749-54.

28. Wallace C, Reiber GE, LeMaster J, Smith DG, Sullivan K, Hayes S, et al. Incidence of falls, risk factors for falls, and fall-related fractures in individuals with diabetes and a prior foot ulcer. Diabetes Care. 2002;25:1983-6.

29. Biver E, Salliot C, Combescure C, Gossec L, Hardouin P, Legroux-Gerot I, et al. Influence of adipokines and ghrelin on bone mineral density and fracture risk: a systematic review and meta-analysis. J Clin Endocrinol Metab. 2011;96:2703-13.

30. Duque G. Bone and fat connection in aging bone. Curr Opin Rheumatol. 2008;20:429-34.

31. Griffin TM, Guilak F. Why is obesity associated with osteoarthritis? Insights from mouse models of obesity. Biorheology. 2008;45:387-98.

32. Sornay-Rendu E, Boutroy S, Vilayphiou N, Claustrat B, Chapurlat RD. In obese postmenopausal women, bone microarchitecture and strength are not commensurate to greater body weight: the Os des Femmes de Lyon (OFELY) study. J Bone Miner Res. 2013;28: 1679-87.

33. Hu WW, Zhang $\mathrm{H}$, Wang $\mathrm{C}$, Gu JM, Yue H, Ke YH, et al. Lean mass predicts hip geometry and bone mineral density in chinese men and women and age comparisons of body composition. J Clin Densitom. 2012;15:434-42.

34. Bergot C, Bousson V, Meunier A, Laval-Jeantet M, Laredo JD. Hip fracture risk and proximal femur geometry from DXA scans. Osteoporos Int. 2002;13:542-50.

35. Law CG, Brookmeyer R. Effects of mid-point imputation on the analysis of doubly censored data. Stat Med. 1992;11:1569-78.

36. Gilsanz V, Chalfant J, Mo AO, Lee DC, Dorey FJ, Mittelman SD. Reciprocal relations of subcutaneous and visceral fat to bone structure and strength. J Clin Endocrinol Metab. 2009;94: 3387-93.

37. Cummings SR, Black DM, Nevitt MC, Browner W, Cauley J, Ensrud K, et al. Bone density at various sites for prediction of hip fractures. The Study of Osteoporotic Fractures Research Group. Lancet. 1993;341: 72-5. 